M. Münch

C. Cajochen

A. Wirz-Justice

\title{
Schlaf und zirkadiane Rhythmik im Alter
}

\section{Sleep and circadian rhythms in ageing}

Zusammenfassung Mit steigendem Alter nimmt die nächtliche Schlafkonsolidierung ab, kurze Nickerchen über den Tag nehmen $\mathrm{zu}$, und die Schlafzeit verschiebt sich in frühere Stunden. Die Schlafregulation hängt von der
Diese Forschung wurde ermöglicht durch finanzielle Unterstützung des Schweizerischen Nationalfonds, Projektnummern: 3130-054991.98 und 3100-055385.98 zu Gunsten von CC sowie der Velux-Stifung, $\mathrm{CH}$

Mirjam Münch MS (

Christian Cajochen Ph.D.

Anna Wirz-Justice Ph.D.

Zentrum der Chronobiologie

Universitäre Psychiatrische Kliniken

Wilhelm-Klein-Strasse 27

4025 Basel, Schweiz
Interaktion zwischen einem zirkadianen Schrittmacher (biologische Uhr) und dem Schlafhomöostaten (je länger die Wachphase, desto größer der Schlafdruck) ab. Wir konnten an gesunden älteren Personen zeigen, dass sich die Amplitude zirkadianer Rhythmen (z.B. die Melatonin-Sekretion) und die Tiefschlafdauer verringert. Gleichzeitig nimmt die Müdigkeit am Nachmittag zu, wie auch die Tendenz - im Gegensatz zu jüngeren Personen - am frühen Abend einzuschlafen. Da Licht der Hauptzeitgeber ist, um die biologische Uhr zu stabilisieren, brauchen ältere Menschen tagsüber und am Abend genügend Licht und sollten während des Tages keine oder nur kurze Nickerchen machen, um in der Folge den Schlaf in der Nacht zu verbessern.

\section{Schlüsselwörter}

Zirkadiane Rhythmik Schlafhomöostase - Melatonin Nickerchen - Licht
Summary With age, the consolidation of nocturnal sleep decreases, daytime napping increases, and sleep occurs earlier. Sleep regulation is dependent on the interaction between a circadian pacemaker (biological clock) and the sleep homeostat (sleep pressure increasing with duration of time awake). We have shown that in the healthy elderly, the amplitude of circadian rhythms (e.g. melatonin secretion) declines, as does slow wave sleep, parallel with an increase in afternoon sleepiness and a tendency to fall asleep in the early evening when younger subjects do not. Light is the major zeitgeber to stabilise the biological clock: older subjects require sufficient light exposure during daytime and in the evening, and should take no or only brief naps during the day to improve sleep.

Key words Circadian system sleep homeostat - melatonin naps - light
Ein gesunder Mensch durchläuft während des Lebens unterschiedliche Entwicklungsstadien mit charakteristischen physischen und psychischen Veränderungen, welche sich auch im Schlafverhalten und den zirkadianen Aktivitätsmustern widerspiegeln. Die Regu-

\footnotetext{
${ }^{1}$ zirkadian: von lat.: circa: $=$ ungefähr; dies $=$ der Tag
}

lation des menschlichen Schlaf-Wach-Rhythmus kann theoretisch in zwei miteinander interagierende Prozesse eingeteilt werden: einen homöostatischen ${ }^{2}$ Prozess (S) und einen zirkadianen Prozess (C) [3]. Der homöostatische Prozess reguliert den Anstieg des

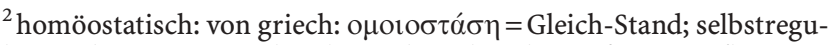
latorisches inneres Milieu bei sich ändernden äußeren Einflüssen
} 
„Schlafdrucks“ (d.h. der Müdigkeit) während des Tages in Abhängigkeit von der verstrichenen Zeit seit dem Aufwachen sowie dessen Abbau in der darauf folgenden Schlafepisode. Der zirkadiane Prozess hingegen beschreibt die Funktion der „inneren Uhr“ mit einer endogenen Periode von durchschnittlich 24,2 Stunden beim Menschen [7]. Da diese Periodendauer länger als 24 Stunden ist, muss sich der Organismus täglich von neuem auf einen 24-Stunden-Tag mit ungefähr 16 Stunden Aktivität und 8 Stunden Schlaf synchronisieren. Hierfür sind äußere „Zeitgeber" notwendig, der wichtigste von ihnen ist das Tageslicht. Bis heute ist noch unbekannt, welches die neurobiologischen Korrelate dieser beiden Prozesse sind, es werden zelluläre, genetische und endokrinologische Faktoren diskutiert [10]. Der Sitz der übergeordneten inneren Uhr befindet sich in den suprachiasmatischen Kernen des Zwischenhirns, welche die Außenwelt (z.B. via Lichteinfall auf die Photorezeptoren der Netzhaut) mit anderen Hirnbereichen sowie den so genannten peripheren Oszillatoren in den einzelnen Körperzellen funktional verbinden und koordinieren. Verletzungen in der Region der suprachiasmatischen Kerne führen zum Verlust eines normalen Tag-Nacht-Rhythmus [2, 6].

Im Alter kann auch bei gesunden Menschen die Anzahl Stunden Schlaf pro Nacht abnehmen. Es treten häufigere Wachepisoden im Schlaf auf, die Bettgehbzw. Aufstehzeiten erfolgen zu einer früheren Uhrzeit und die Tiefschlafphasen werden kürzer [8]. Viele ältere Menschen gönnen sich ein oder mehrere Nickerchen am Tag. Die rasche Zeitumstellung während Schichtarbeit sowie die Anpassung an die jeweils neue lokale Zeit nach einem Flug über mehrere Zeitzonen hinweg fallen älteren Menschen zunehmend schwerer [5]. Um vorbeugende und therapeutische Ansätze in der Praxis anzuwenden, wäre es wichtig zu wissen, welche Prozesse für diese altersbedingten Veränderungen verantwortlich sind und welche Auswirkungen sie auf die Gesundheit haben [10].

In unseren Studien unter konstanten Laborbedingungen, bei welchen die Versuchspersonen entweder sehr viel (alle 2,5 Stunden ein Nickerchen von 1,25 Stunden Dauer) oder gar nicht (40 Stunden Schlafentzug) schlafen durften, konnten wir zeigen, dass nach Schlafentzug ältere gesunde Menschen absolut gerechnet weniger Tiefschlaf hatten als die jüngeren Versuchspersonen. Relativ besehen (d.h. verglichen mit der Ausgangsnacht) „holten“ beide Altersgruppen in der folgenden Erholungsnacht Tiefschlaf „nach“. Gleichzeitig war bei den älteren Versuchspersonen die Aktivität der langsamwelligen Hirnstromkurven des Elektroenzephalogramms (EEG) während der Erholungsnacht in frontalen Hirnbereichen deutlich tiefer [14] als bei jungen Versuchspersonen [4]. Im Experiment mit den insgesamt 11 Nickerchen war der im
Speichel gemessene Melatoninspiegel bei den älteren Versuchspersonen während der Nacht signifikant tiefer als in der jüngeren Altersgruppe (s. Abb. 1A). Die subjektive Müdigkeit hingegen stieg bei den älteren Versuchspersonen am späten Nachmittag und am Abend stärker an als bei den Jungen (s. Abb. 1B). Die totale Schlafzeit (Stadium 1-4, REM-Schlaf) zeigte während der Nickerchen keine signifikanten Unterschiede zwischen den beiden Altersgruppen, wohl aber deren Verteilung über die Zeit. Die jungen Ver-
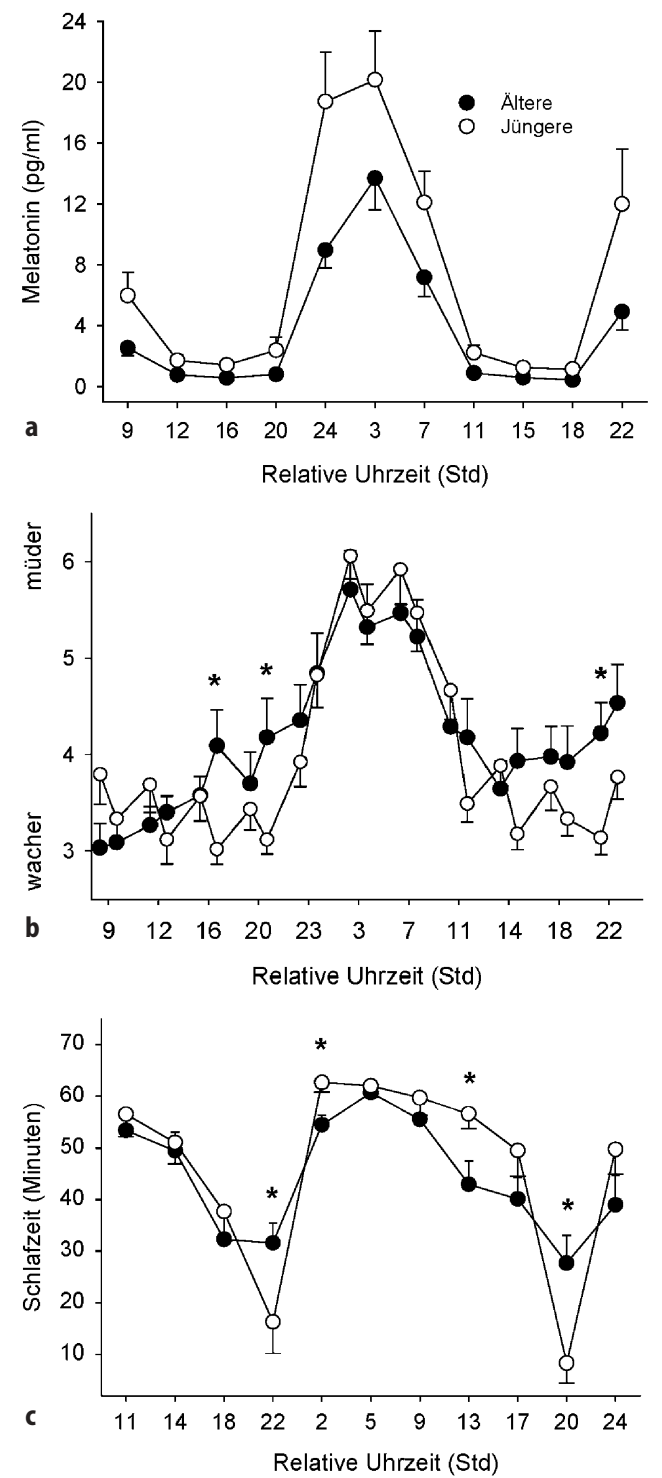

Abb. 1 Resultate aus einem 40-stündigen Protokoll (11 Nickerchen von 1,25 Std. Dauer in 2,5 Std.-Abständen) unter konstanten Laborbedingungen. Jüngere (weiss, $n=17$ ) und ältere Versuchspersonen (schwarz, $n=15$ ). a: Mittlere Melatoninwerte (im Speichel gemessen) b: Subjektiver Müdigkeitsverlauf c: Totale Schlafdauer während 11 Nickerchen. Alle Werte + oder Standardfehler. ${ }^{*} p<0,05$ (Mann-Whitney U Test). [13] mit Erlaubnis von (c) Elsevier Inc. 2005 
suchspersonen konnten zu einer bestimmten Zeit abends praktisch nie einschlafen (s. Abb. 1C). Diese Zeit intensiver Wachheit kurz vor der eigentlichen Bettgehzeit am späten Abend, auch „wake maintenance zone" genannt [11], stellt ein Paradoxon dar, welches mit der Wechselwirkung von Prozess S und C erklärt werden kann. Am Abend kurz vor der Bettgehzeit ist der während des Tages aufgebaute Schlafdruck am größten. Wach zu bleiben zu diesem Zeitpunkt erfordert einen entgegengesetzten Mechanismus, welcher nur durch ein stärkeres „Wachheitssignal“ des zirkadianen Systems erreicht werden kann. In der älteren Versuchsgruppe war diese auffallende Wachheit am Abend stark vermindert, d.h., die meisten Personen konnten auch zu diesem Zeitpunkt schnell einschlafen [13]. Eine mögliche Interpretation für dieses Resultat ist entweder ein altersbedingt niedrigeres zirkadianes Wachheitssignal (Prozess C) am Abend oder aber ein stärkerer Schlafdruck (Prozess S) - oder die Wirkung von beidem. Demnach könnte die Interaktion dieser beiden abgeschwächten Prozesse dazu beitragen, dass gesunde ältere Menschen am Tag und gegen Abend müder sind, ihr Schlaf weniger konsolidiert ist und sie gegen Morgen früher erwachen als Junge.

Ein weiterer wichtiger Faktor für die zirkadiane Rhythmik im Alter stellt die Beleuchtungsstärke des Lichts in der Umgebung dar, welches via Netzhaut das zirkadiane System und somit den Schlaf-Wach-
Rhythmus des Menschen beeinflusst. Sowohl Augenprobleme wie Verhaltensänderungen spielen eine Rolle. So leiden nicht wenige, sonst gesunde ältere Menschen unter Katarakten, Glaskörpertrübungen, Netzhauterkrankungen etc. Hinzu kommt, dass Ältere eher weniger Zeit im Freien verbringen und damit weniger lang dem normalen Tageslicht ausgesetzt sind. Zusammen mit einer unzureichenden Beleuchtung, wie sie leider nur allzu oft zu Hause oder in geriatrischen Institutionen anzutreffen ist, wirkt sich dies als ein deutlich schwächerer Zeitgeber aus. Studien in Altersheimen haben diesen Lichtmangel klar bewiesen, und therapeutische Ansätze mit helleren Aufenthaltsräumen haben nicht nur den SchlafWach-Rhythmus verbessern können, sondern auch den nächtlichen endogenen Melatoninspiegel erhöht $[12,15,16]$. Dies erhält eine besondere Bedeutung bei dementen Patienten, welche sehr häufig unter Schlafstörungen und/oder einer Tag-Nacht-Umkehr leiden $[1,9]$.

Als Grundsätze für gesunde ältere Menschen könnten demnach gelten:

Mehr Licht: Verbringen Sie mindestens 30 Minuten pro Tag am Tageslicht.

Wenn Sie ein Nickerchen am Tag machen, sollte dies nicht länger als 30-60 Minuten dauern.

Sorgen Sie für ausreichend körperliche Aktivität (30 Minuten spazieren).

Gehen Sie nicht zu früh zu Bett.

\section{Literatur}

1. Ancoli-Israel S, Martin JL, Kripke DF et al (2002) Effect of light treatment on sleep and circadian rhythms. JAGS 50:282-289

2. Bloch K, Brack T, Wirz-Justice A (2005) Transient short free-running circadian rhythm in a case of aneurysm near the suprachiasmatic nuclei. J Neurol Neurosurg Psychiatry 76:1178-1180

3. Borbély AA (1982) A two process model of sleep regulation. Human Neurobiol 1:195-204

4. Cajochen C, Foy R, Dijk DJ (1999) Frontal predominance of a relative increase in sleep delta and theta EEG activity after sleep loss in humans. Sleep Res Online 2:65-69

5. Campbell, S (1995) Effects of timed bright-light exposure on shift-work adaptation in middle-aged subjects. Sleep 18:408-416

6. Cohen RA, Albers HE (1991) Disruption of the human circadian and cognitive regulation following a discrete hypothalamic lesion: a case study. Neurol 41:726-729
7. Czeisler CA, Duffy JF, Shanahan TL et al (1999) Stability, precision, and near-24-hour period of the human circadian pacemaker. Science 284:2177-2181

8. Dijk DJ, Duffy JF, Czeisler CA (2000) Contribution of circadian physiology and sleep homeostasis to age-related changes in human sleep. Chronobiol Int 17:285-311

9. Fontana Gasio P, Kräuchi K, Cajochen C et al (2003) Dawn-dusk simulation light therapy of disturbed circadian rest-activity cycles in demented elderly. Exp Gerontol 38:207-216

10. Foster RG, Wulff K (2005) The Rhythm of rest and excess. Nat Rev Neurosci 6:407-414

11. Lavie P (1986) Ultrashort sleep-waking schedule III. "Gates" and "forbidden zones" for sleep. Electroencephalogr Clin Neurophysiol 63:414425
12. Mishima K, Okawa M, Shimitzu T et al (2001) Diminished melatonin secretion in the elderly caused by insufficient environmental illumination. J Clin Endocrinol Metab 86:129-134

13. Münch $\mathrm{M}$, Knoblauch V, Blatter K et al (2005) Age-related attenuation of the evening circadian arousal signal in humans. Neurobiol Aging. Epub 18 April. DOI: 10.1016/j.neurobiolaging. 2005.03.004

14. Münch $\mathrm{M}$, Knoblauch V, Blatter $\mathrm{K}$ et al (2004) The frontal predominance in human EEG delta activity after sleep loss decreases with age. Eur J Neurosci 20:1402-1410

15. Shochat T, Martin J, Marler $M$ et al (2000) Illumination levels in nursing home patients: effects on sleep and activity rhythms. J Sleep Res 9:373379

16. Van Someren EJ, Riemersma RF, Swaab DF (2002) Functional plasticity of the circadian timing system in old age: light exposure. Prog Brain Res 138:205-231 